\title{
Mudanças climáticas e impactos na necessidade hídrica das culturas perenes na Bacia do Jaguaribe, no Estado do Ceará
}

\author{
Rubens Sonsol Gondim(1), Marco Aurélio Holanda de Castro(2), Sílvio Roberto de Medeiros Evangelista(3), \\ Adunias dos Santos Teixeira(2) e Sérgio César de França Fuck Júnior ${ }^{(1)}$
}

(1)Embrapa Agroindústria Tropical, Rua Dra. Sara Mesquita, n 2270, CEP 60511-110 Fortaleza, CE. E-mail: rubens@cnpat.embrapa.br, sergiofuck@cnpat.embrapa.br (2)Universidade Federal do Ceará, Avenida Mister Hull, s/n, Blocos 713 e 804, CEP 60455-760 Fortaleza, CE. E-mail: marco@ufc.br, adunias@ufc.br ${ }^{(3)}$ Embrapa Informática Agropecuária, Caixa Postal 6041, CEP $13083-886$ Campinas, SP. E-mail: silvio@cnptia.embrapa.br

Resumo - O objetivo deste trabalho foi avaliar os impactos das mudanças climáticas na demanda de água para irrigação de culturas perenes, na Bacia do Jaguaribe, no Estado do Ceará. Foi empregado o sistema integrado de modelagem regional PRECIS ("Providing Regional Climates for Impact Studies"), e aplicado o método de redução de escala de bacia hidrográfica, com as condições de contorno do modelo climático regional (HadRM3P). Foi utilizado um conjunto de climatologia de base do modelo de 1961 a 1990 e de projeções climáticas futuras. As coordenadas geográficas da região em estudo foram consideradas para interpolação num sistema de informação geográfica. A evapotranspiração de referência foi estimada por meio de dados da temperatura média mensal. As mudanças climáticas projetadas aumentaram a demanda projetada de água para irrigação, porque a evapotranspiração foi estimada para aumentos de 3,1 a 2,2\% e a precipitação pluvial foi estimada para diminuições de 30,9 a $37,3 \%$. O aumento da necessidade hídrica foi estimada em $32,9 \%$ a $43,9 \%$, para o ano de 2040, conforme o cenário analisado.

Termos para indexação: evapotranspiração, irrigação, SIG.

\section{Climate change and impacts on water requirement of permanent crops in the Jaguaribe Basin, Ceará, Brazil}

\begin{abstract}
The aim of this study was to estimate climate change impacts on irrigation water demand for permanent crops. The PRECIS (Providing Regional Climates for Impact Studies) system was applied, and downscaling techniques were used at the river basin level, with the boundary conditions of the regional climate model (HadRM3P). A climate data set was generated for 1961 to 1990 (baseline) and for future climate projections. The regional geographical coordinates were considered for interpolation in a georeferenced coordinated system. The reference evapotranspiration was estimated through data of monthly average temperature. Projected climate change increased projected irrigation water demand, because evapotranspiration was estimated to increase by 3.1 to $2.2 \%$ and rainfall was estimated to decrease by 30.9 to $37.3 \%$. The 2040 water need was estimated to increase by $32.9 \%$ to $43.9 \%$, according to the analyzed scenario.
\end{abstract}

Index terms: evapotranspiration, irrigation, GIS.

\section{Introdução}

Os modelos de circulação global, geralmente utilizados para simular o clima presente e a projeção climática futura, com forçantes de gases de efeito estufa e aerossóis, dividem a atmosfera e o oceano em grades de resolução horizontal de 2 a $4^{\circ}$ de latitude e longitude, com 10 a 20 camadas na vertical. Em geral, simulam processos na escala global ou continental em detalhe e provêem uma representação de precisão razoável da média climática planetária. Por outro lado, esses métodos são incapazes de representar as características locais (Dibike \& Coulibaly, 2005).

Binder (2006) recomenda detalhar os cenários disponíveis das mudanças climáticas para a escala de bacia hidrográfica, utilizando-se, também, modelos hidrológicos, a fim de se estudar impactos das mudanças nos recursos hídricos. Essa redução de escala dinâmica inclui o aninhamento de um modelo de circulação regional a uma resolução mais grosseira de um modelo 
de circulação global. O modelo de circulação regional utiliza o modelo de circulação global para definir as condições de contorno atmosféricas, variáveis com o tempo, em torno de um domínio finito (Wilby et al., 2002).

Schlenker et al. (2007), ao analisar o impacto da mudança climática na agricultura irrigada na Califórnia, concluíram que, com aplicação de uma escala geográfica menor, o escoamento superficial durante a maior parte da estação de cultivo sofre decréscimo, com a possibilidade de modesto aumento na precipitação, o que resulta em redução na disponibilidade de água quando ela é mais necessária, aumento da demanda para irrigação, aumento da pressão sobre os rios e sobre os sistemas de água subterrânea.

Döll \& Hauschild (2002) analisaram os impactos potenciais das mudanças climáticas de longo prazo nas necessidades hídricas para irrigação nos estados do Ceará e do Piauí. Os autores compararam as necessidades hídricas das culturas em diferentes cenários derivados da redução de escala dos modelos climáticos ECHAM4 (Roeckner et al., 1996) e HadCM2 (Johns et al., 1997). Observaram que a precipitação projetada reduziu ligeiramente no caso do ECHAM4, e que cenários futuros resultam em redução de demanda de $48 \%$ para a maioria dos municípios com irrigação (até $6,5 \mathrm{~mm}$ por ano) e resultam em aumento de demanda de apenas $4 \%$ (menos de 0,3 mm por ano). Com o HadCM2, as reduções das necessidades para irrigação foram ainda maiores. Por outro lado, os autores alertaram não ter modelado a variedade de culturas e consideraram taxa fixa de eficiência de uso da água de 0,6. Concluíram que o modelo possivelmente subestimou o uso da água de irrigação em razão da estimativa conservadora de áreas irrigadas, da negligência da variabilidade de culturas e de valor elevado para eficiência de uso da água de irrigação.

Krol et al. (2006) e Krol \& Bronstert (2007) aplicaram o modelo "Semi-arid Integrated Model" - SIM (Modelo Integrado do Semi-Árido), também sobre os estados do Ceará e do Piauí, com cenários climáticos futuros, resultantes da técnica estatística e de tendências climáticas dos Modelos de Circulação Global: ECHAM4 e HadCM2.

Esses autores observaram que projeções para precipitação sobre a região (2070/2090, em relação a
1961/1990) divergiram, sendo estimado 50\% menos quando utilizado o modelo ECHAM4 e $21 \%$ mais com o modelo HadCM2.

A aplicação de modelo climático regional permite a avaliação de impactos em áreas menores, e a melhoria na resolução espacial permite a análise mais detalhada dos impactos locais.

O objetivo deste trabalho foi avaliar os impactos das mudanças climáticas na necessidade hídrica das culturas perenes na Bacia do rio Jaguaribe, no Estado do Ceará, considerando a variedade das culturas irrigadas, a eficiência dos sistemas de irrigação e os cenários de mudanças climáticas.

\section{Material e Métodos}

A área do estudo foi delimitada entre $4^{\circ} 39^{\prime} 30^{\prime \prime} \mathrm{e}$ $5^{\circ} 40^{\prime} 0^{\prime \prime} \mathrm{S}$ e $37^{\circ} 35^{\prime} 30^{\prime \prime}$ e $38^{\circ} 27^{\prime} 0^{\prime \prime} \mathrm{W}$, com superfície de $6.415,10 \mathrm{~km}^{2}$. Foi identificada a tipologia da agricultura irrigada praticada na região (culturas, tecnologias empregadas e áreas), com todas as áreas de irrigação difusa e o Distrito de Irrigação Jaguaribe-Apodi (Dija) que captam água diretamente no rio. O trabalho abrangeu um trecho de $160 \mathrm{~km}$ do rio Jaguaribe e incluiu os municípios de Alto Santo, São João do Jaguaribe, Tabuleiro do Norte, Limoeiro do Norte, Quixeré, Jaguaruana, Russas e Itaiçaba, no Estado do Ceará.

Foi utilizado o sistema integrado de modelagem climática regional PRECIS ("Providing Regional Climates for Impacts Studies"), versão 1.2 (Jones et al., 2004), desenvolvido no Hadley Centre, Inglaterra, com as condições de contorno do modelo climático regional HadRM3P, o qual foi implementado no Brasil pelo Centro de Previsão de Tempo e Estudos Climáticos (Cptec), do Instituto Nacional de Pesquisas Espaciais (Inpe). Sua dinâmica é baseada no HadRM3, que é uma versão mais recente da componente atmosférica do modelo de circulação global HadCM3 (Johns et al., 2003).

O modelo regional HadRM3 possui resolução horizontal de $50 \mathrm{~km}$ com 19 níveis na vertical (da superfície até $30 \mathrm{~km}$ na estratosfera) e quatro níveis no solo. A resolução espacial é de $0,44 \times 0,44^{\circ}$ (latitude $\mathrm{x}$ longitude), o que corresponde a uma grade aproximada de 50x50 km. Para fins de mudança climática, o modelo usa uma representação do ciclo de sulfato e de alguns outros aerossóis. 
Nakicenovic et al. (2000) definiram uma família de cenários básicos que foram classificados da seguinte forma: A1: baixo crescimento populacional, crescimento do PIB muito elevado, elevado consumo de energia, baixa mudança no uso da terra, alta a média disponibilidade de recursos, rápido desenvolvimento tecnológico, energia fóssil e balanceada; A2: alto crescimento populacional, médio crescimento do PIB, elevado consumo de energia, média a alta mudança no uso da terra, baixa disponibilidade de recursos, lento desenvolvimento tecnológico, energia regional; B1: baixo crescimento populacional, elevado crescimento do PIB, baixo consumo de energia, alta mudança no uso da terra, baixa disponibilidade de recursos, médio desenvolvimento tecnológico, eficiência e introdução de tecnologias limpas; B2: médio crescimento populacional, médio crescimento do PIB, médio consumo de energia, média mudança no uso da terra, média disponibilidade de recursos, médio desenvolvimento tecnológico, dinâmica energética como a usual.

As mudanças climáticas dependem das forçantes que atuam nas emissões de dióxido de carbono e de aerossóis para a atmosfera, tais como: população, intensidade de emissão de carbono das fontes de energia, emissões sulfúricas, modalidade de crescimento econômico e desenvolvimento tecnológico. Os cenários A1 e B1 possuem as mesmas projeções populacionais, assumindo-se baixas taxas de fertilidade e de mortalidade. $\mathrm{O}$ cenário A2 assume alta fertilidade e alta mortalidade e o cenário B2 é intermediário. Optou-se, então, por simular os cenários A2 e B2 que projetam para 2040, uma população global entre 8,93 e 10,71 bilhões de habitantes (Nakicenovic et al., 2000).

Foi utilizada neste trabalho uma metodologia similar à aplicada pelos autores Knox et al. (1997), Tsanis \& Naoum (2003) e Victoria et al. (2005), para estimar as necessidades de água da agricultura irrigada em bacia hidrográfica. Foi utilizado um Sistema de Informação Geográfica (SIG) para colher informação espacial de dados meteorológicos e de distribuição de produtores irrigantes, a fim de se estimar a demanda de irrigação, pela incorporação da variabilidade espacial e temporal das variáveis de demanda de água de irrigação (Fortes et al., 2005).

O horizonte temporal para avaliação dos impactos das mudanças climáticas considerado foi o ano de 2040. Os dados de saída do modelo foram interpolados, e considerou-se a latitude/longitude, por meio de geoestatística (krigagem), associada a um SIG e posterior elaboração de mapas temáticos. A distância considerada não é geométrica e sim estatística, o que distingue a krigagem do método do inverso do quadrado da distância (Díaz et al., 2007; Silva et al., 2007).

No presente trabalho, assumiu-se que, no início das irrigações, não houve deficit de umidade no solo, conforme Díaz et al. (2007). Em razão da freqüência de irrigação requerida para uma cultura sob determinado clima depender da quantidade de água armazenada no solo (Bernado et al., 2005), considerou-se o solo como um reservatório de água que influencia o manejo e a freqüência de irrigação, e não afeta o potencial mensal demandado pelas culturas.

A necessidade hídrica líquida das culturas (NHL) pode ser definida como a quantidade de água que as plantas cultivadas devem receber para se desenvolverem sem estresse hídrico (Fischer et al., 2007), e é modelada pela seguinte expressão:

$\mathrm{NHL}=\mathrm{EToKcfc}-\mathrm{ppt}_{\text {efetiva }}$

em que: NHL, é a necessidade hídrica líquida (mm); ETo, evapotranspiração de referência $(\mathrm{mm})$; Kc, coeficiente de cultivo (adimensional); fc, fator de cobertura da cultura (adimensional); e ppt $_{\text {efetiva, }}$ precipitação efetiva $(\mathrm{mm})$.

A FAO recomendou a adoção do método combinado de Penman-Monteith, que ficou conhecido como o método FAO Penman-Monteith, e é padrão mundial para cálculo das necessidades hídricas das culturas (Allen et al., 1998). Entretanto, em situações em que se disponha apenas de temperatura do ar, como o modelo regional HadRM3P, a evapotranspiração de referência (ETo) pode ser estimada pela equação de Blaney-Criddle (Doorenbos \& Pruit, 1977), que apesar de ser reconhecida por subestimar a evapotranspiração, conforme Samani \& Pessarakli (1986), considerou-se o alto grau de correlação (0,83 para região semi-árida) existente entre as duas equações (Mohan, 1991).

O levantamento das áreas irrigadas na bacia permitiu conhecer a tipologia da agricultura irrigada praticada na região que apresenta uma combinação de culturas perenes. Estimou-se, então, um coeficiente de cultivo $(\mathrm{Kc})$ ponderado pela área ocupada por cada cultura perene em estádio produtivo, que resultou num $\mathrm{Kc}$ de 0,96 e uma eficiência de aplicação ponderada pela área ocupada por cada método de irrigação, de 0,67, superior, portanto, ao considerado por Döll \& Hauschild 
(2002) (Tabelas 1 e 2). Essa ponderação, equivale matematicamente à soma da evapotranspiração das culturas estimada individualmente pelos coeficientes de cultivos de cada cultura irrigada.

$\mathrm{O}$ fator de cobertura das culturas $(\mathrm{fc})$ considera a superfície foliar que evapotranspira como uma fração da área cultivada, que é função da espécie irrigada e do espaçamento adotado, e que varia com o seu desenvolvimento (Allen et al., 1998). Neste trabalho, foi considerado para culturas permanentes em estágio de máximo desenvolvimento como igual a 0,8 , ou seja, $80 \%$ da área irrigada ocupada por cobertura vegetal. Foi necessário atribuir esse valor, com base em experiência de campo, uma vez que na literatura não se identificou dados para a grande maioria das culturas existentes, o que é uma incerteza na precisão do volume de água demandado e estimado pelo modelo, o que não influencia, entretanto, na tendência de incremento ou de redução de demanda de água, ocasionada pelas mudanças climáticas.

Em razão da NHL ser igual à evapotranspiração da cultura (ETc), a necessidade hídrica bruta (NHB) é igual à NHL dividida pela eficiência de irrigação, para que sejam consideradas as perdas por evaporação, escoamento superficial e percolação profunda. A partir das necessidades de irrigação líquida e da eficiência final de irrigação, foram estimadas as necessidades hídricas brutas (NHB): $\mathrm{NHB}=10(\mathrm{NHL} / \mathrm{Ea})$ em que: Ea é a eficiência de aplicação (decimal); NHB, a necessidade hídrica bruta $\left(\mathrm{m}^{3} \mathrm{ha}^{-1}\right) ; 10$, fator de conversão de mm para $\mathrm{m}^{3} \mathrm{ha}^{-1}$.

Foram considerados os valores de eficiência de aplicação (Ea) alcançados pelos produtores irrigantes da região, obtidos nos relatos de Costa \& Souza (2006) e Nunes (2006), e definiu-se uma média ponderada pela área ocupada por cada método de irrigação.

\section{Resultados e Discussão}

O próprio modelo climático adotado permitiu projetar mensalmente aumentos na temperatura e, conseqüentemente, elevação acumulada na evapotranspiração de referência (ETo) anual, em relação ao período de 1960 a 1990 , de $3,1 \%$ e de $2,2 \%$, e redução na precipitação pluvial acumulada anual de $30,9 \%$ e de $37,3 \%$ em 2040, para os cenários A2 e B2, respectivamente.

Foi projetada uma elevação da necessidade hídrica bruta, média anual, em 2040, relativa às condições iniciais (climatologia de base), de 4.070 e $5.434 \mathrm{~m}^{3} \mathrm{ha}^{-1}$ (32,9 e 43,9\%), para os cenários A2 e B2, respectivamente (Tabela 3 ).

Embora os percentuais estimados nopresente trabalho tenham sido maiores, a elevação das necessidades de água para irrigação pelas mudanças climáticas também foi prevista por Silva et al. (2007), que aplicaram o modelo HadCM3 (resolução de 300x300 km) no Sri Lanka, numa resolução local de 16x16 km. Os autores

Tabela 1. Tipologia da agricultura irrigada e coeficiente de cultivo médio ponderado pela área para as culturas perenes.

\begin{tabular}{|c|c|c|c|c|c|c|c|c|}
\hline Culturas & Inundação (ha) & Sulco (ha) & Aspersão (ha) & Localizada (ha) & Pivô (ha) & Total (A) (ha) & $\mathrm{Kc}$ & $\mathrm{Kc} \times \mathrm{A}$ \\
\hline Açaí & $-(1)$ & - & - & 9,00 & - & 9,00 & 1,00 & 9,00 \\
\hline Acerola & - & - & - & 78,00 & - & 78,00 & 1,00 & 78,00 \\
\hline Anonáceas & 1,50 & 4,20 & - & 96,75 & 15,75 & 118,20 & 0,90 & 106,38 \\
\hline Banana & 92,80 & 61,45 & 5,30 & 748,05 & 17,75 & 925,35 & 1,20 & $1.110,42$ \\
\hline Caju & - & 0,50 & 0,75 & 17,75 & - & 19,00 & 0,65 & 12,35 \\
\hline Cana & 14,30 & - & - & - & 25,00 & 39,30 & 1,25 & 49,13 \\
\hline Capim & 207,05 & 56,42 & 44,25 & 3,00 & 225,00 & 535,72 & 0,75 & 401,79 \\
\hline Citros & 53,75 & 44,35 & - & 3,50 & - & 101,60 & 0,80 & 81,28 \\
\hline Coco & 2,45 & 1,20 & 1,80 & 93,50 & - & 98,95 & 1,00 & 98,95 \\
\hline Figo & - & - & - & 0,50 & - & 0,50 & 1,00 & 0,50 \\
\hline Forragem & 11,50 & 2,25 & 4,50 & - & - & 18,25 & 0,75 & 13,69 \\
\hline Goiaba & 8,65 & 4,20 & 1,00 & 113,70 & - & 127,55 & 0,63 & 80,36 \\
\hline Mamão & 0,50 & - & - & 4,70 & - & 5,20 & 0,80 & 4,16 \\
\hline Manga & - & 0,90 & - & 135,00 & - & 135,90 & 0,71 & 96,49 \\
\hline Maracujá & - & - & 0,50 & 0,20 & - & 0,70 & 0,69 & 0,48 \\
\hline Ornamentais & - & - & 0,50 & 4,00 & - & 4,50 & 1,00 & 4,50 \\
\hline Sapoti & - & 0,50 & - & 7,50 & - & 8,00 & 0,70 & 5,60 \\
\hline Uva & - & - & - & 19,50 & - & 19,50 & 0,60 & 11,70 \\
\hline Total & 392,50 & 175,97 & 58,60 & $1.334,65$ & 283,50 & $2.245,22$ & 0,96 & $2.164,77$ \\
\hline
\end{tabular}

${ }^{(1)}$ Refere-se a culturas não irrigadas pelo método correspondente. Fonte: elaborada a partir de dados da Companhia de Gestão de Recursos Hídricos do Ceará (Cogerh, 2008). 
previram aumento na demanda de água para irrigação de arroz em 2050, de 23 e 13\%, em média, para os cenários A2 e B2, respectivamente. Isto ocorreu principalmente em razão da redução da precipitação (-17 e $-9 \%$, cenários A2 e B2, respectivamente) durante a época de irrigação - embora tenha sido previsto um aumento da precipitação total anual pelo modelo -, combinada com um aumento da evapotranspiração (2 e 1\% para os cenários $\mathrm{A} 2$ e $\mathrm{B} 2$, respectivamente), decorrente do aumento da temperatura $\left(1,4\right.$ e $1,0^{\circ} \mathrm{C}$ para os cenários $\mathrm{A} 2$ e $\mathrm{B} 2$, respectivamente).

A mesma tendência foi verificada por Díaz et al. (2007) que estimaram para 2050, na bacia de Gualdalquivir, Espanha, com o modelo HadCM3, elevações na média das necessidades hídricas de aproximadamente 19,3 e 16,3\%, nos cenários A2 e $\mathrm{B} 2$, respectivamente, causadas principalmente pelo aumento da evapotranspiração $(8,6$ e 7,7\%, cenários A2 e B2, respectivamente) e pela redução da precipitação na época de plantio $(-6,7$ e $-0,5 \%$, para os cenários A2 e B2, respectivamente), acompanhadas de um prolongamento do período anual de irrigação.

O cenário A2 estabelece para 2040 emissões cumulativas de $\mathrm{CO}_{2}$, de $530,7 \mathrm{Gt}$, e o cenário $\mathrm{B} 2$, de 446,0 Gt (Nakicenovic et al., 2000), sendo o A2 mais crítico em termos de mudanças climáticas. Neste trabalho, o aumento das necessidades hídricas apresentou-se maior no cenário B2. Isso pode ser atribuído, principalmente, ao fato de o modelo projetar uma redução da precipitação maior naquele cenário (37,3\%), em relação ao A2 (30,9\%), e uma elevação na evapotranspiração decorrente do aumento da temperatura mais modesta $(2,2$ e $3,1 \%$, respectivamente), quando comparada com as reduções na precipitação.

A mesma situação foi verificada por Medeiros (2003), que avaliou os impactos das mudanças climáticas com o modelo estocástico de precipitação, para transferir as alterações na precipitação, gerada por

Tabela 2. Determinação da eficiência média ponderada pela área de cada método de irrigação.

\begin{tabular}{lccr}
\hline Sistema de irrigação & Área em ha $(\mathrm{A})$ & Eficiência $(\mathrm{B})$ & $\mathrm{AxB}$ \\
\hline Inundação & 392,50 & 0,52 & 204,10 \\
Sulco & 175,97 & 0,54 & 95,02 \\
Aspersão & 58,60 & 0,64 & 37,50 \\
Localizada difusa & $1.334,65$ & 0,74 & 987,64 \\
Pivô & 283,50 & 0,65 & 184,28 \\
\hline Total & $2.245,22$ & & $1.508,54$ \\
\hline Média ponderada & & 0,67 & \\
\hline
\end{tabular}

Fonte: adaptado de Costa \& Souza (2006) e Nunes (2006). um modelo de circulação global da atmosfera, na bacia do rio Paraguaçu, no Estado da Bahia, e concluiu que os impactos hidrológicos na bacia do estudo eram mais fortemente controlados pelas reduções na precipitação do que pelos acréscimos da temperatura.

Para culturas tropicais perenes em clima semi-árido, os aumentos nas necessidades de irrigação são agravados, pois pode também ocorrer prolongamento do período anual de irrigação decorrente da redução na precipitação e não do encurtamento da estação fria, como ocorre em climas temperados (Fischer et al., 2007. Esses autores consideram que a extensão do período de cultivo (ciclo), em conseqüência das mudanças climáticas, pode ter pequenas alterações nos trópicos, e que mudanças na precipitação, combinadas com elevação na demanda da evapotranspiração, aumentam as necessidades hídricas da irrigação.

Enquanto a elevação de $\mathrm{CO}_{2}$ apenas tende a acelerar o crescimento das plantas e a produtividade das culturas, temperaturas mais elevadas e mudanças no regime pluviométrico podem tanto beneficiar quanto prejudicar os sistemas agrícolas. Aumento na concentração de $\mathrm{CO}_{2}$ pode induzir o fechamento dos estômatos das plantas e, assim, reduzir perdas de água por transpiração, conforme sugere Tubiello \& Ewert (2002).

Quando a transpiração é reduzida, a perda de calor pelas folhas é mais lenta e a temperatura delas se eleva. O estresse hídrico nas plantas aumenta, em razão da baixa umidade, pelas temperaturas altas, ventos fortes e alta intensidade luminosa. Segundo Eitzinger et al. (2003), incertezas permanecem em razão da falta de conhecimento de modelos de simulação de plantas,

Diante das mudanças climáticas na região, torna-se importante reduzir as incertezas de como a temperatura e, especialmente, a precipitação deverão se comportar, e de qual será o efeito do aumento da concentração de $\mathrm{CO}_{2}$ na fisiologia das plantas, por meio do aperfeiçoamento de modelos, a fim de se planejar medidas adaptativas para os produtores irrigantes, tais como: melhorias de eficiência na aplicação da água, escolha de métodos de

Tabela 3. Necessidade hídrica bruta anual $\left(\mathrm{m}^{3} \mathrm{ha}^{-1}\right)$ para as culturas perenes, no período de 1961 a 1990 e em 2040, nos cenários A2 e B2.

\begin{tabular}{lccc}
\hline Necessidade hídrica & $1961-1990$ & $2040 \mathrm{~A} 2$ & $2040 \mathrm{~B} 2$ \\
\hline Mínimo & 0 & 2.535 & 2.008 \\
Máximo & 16.515 & 19.673 & 20.830 \\
Média & 12.380 & 16.450 & 17.814 \\
Desvio padrão & 4.342 & 2.881 & 3.103 \\
\hline
\end{tabular}


irrigação, adaptação no padrão de cultivo e seleção de culturas que consumam menos água.

Outro aspecto a se considerar é a melhoria da estimativa da demanda de água para irrigação, utilizando a evapotranspiração de referência estimada pelo método FAO Penman-Monteith ou, na impossibilidade, por métodos que consideram a radiação (Allen et al., 1998).

Os mapas temáticos das necessidades hídricas brutas totais anuais para 1961/1990 e para o ano de 2040, cenários A2 e B2, estão apresentados nas (Figuras 1, 2 e 3). Mesmo em uma área menor (sub-bacia hidrográfica) que as estudadas por Díaz et al. (2007) e Silva et al. (2007), na Espanha e no Sri Lanka, respectivamente, observa-se também variabilidade espacial dos resultados. A demanda de água eleva-se à medida que se dirige para o interior do semi-árido, e apresenta-se menor em localidades mais próximas do litoral, para todas as escalas temporais estudadas.

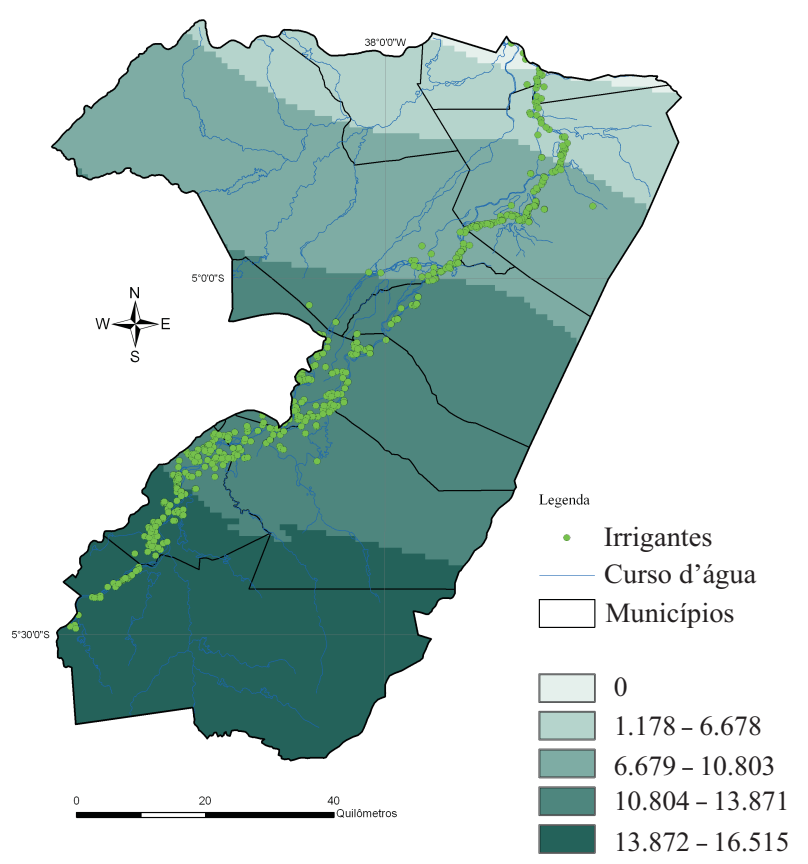

Figura 1. Mapa temático da necessidade hídrica bruta $\left(\mathrm{m}^{3} \mathrm{ha}^{-1}\right)$ anual no período de 1961 a 1990.

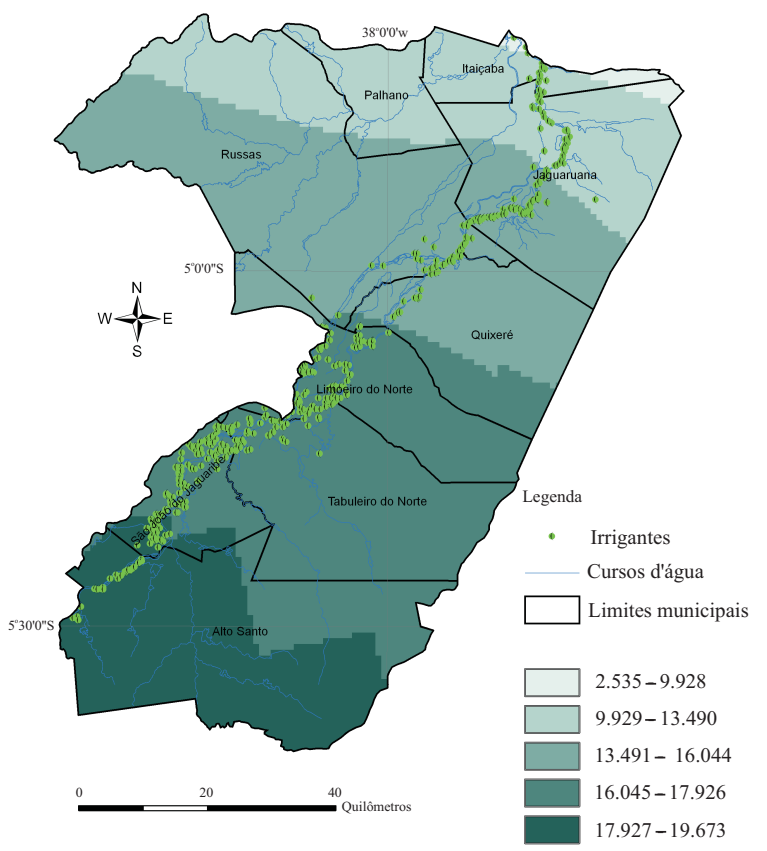

Figura 2. Mapa temático da necessidade hídrica bruta $\left(\mathrm{m}^{3} \mathrm{ha}^{-1}\right)$ anual em 2040, no cenário A2.

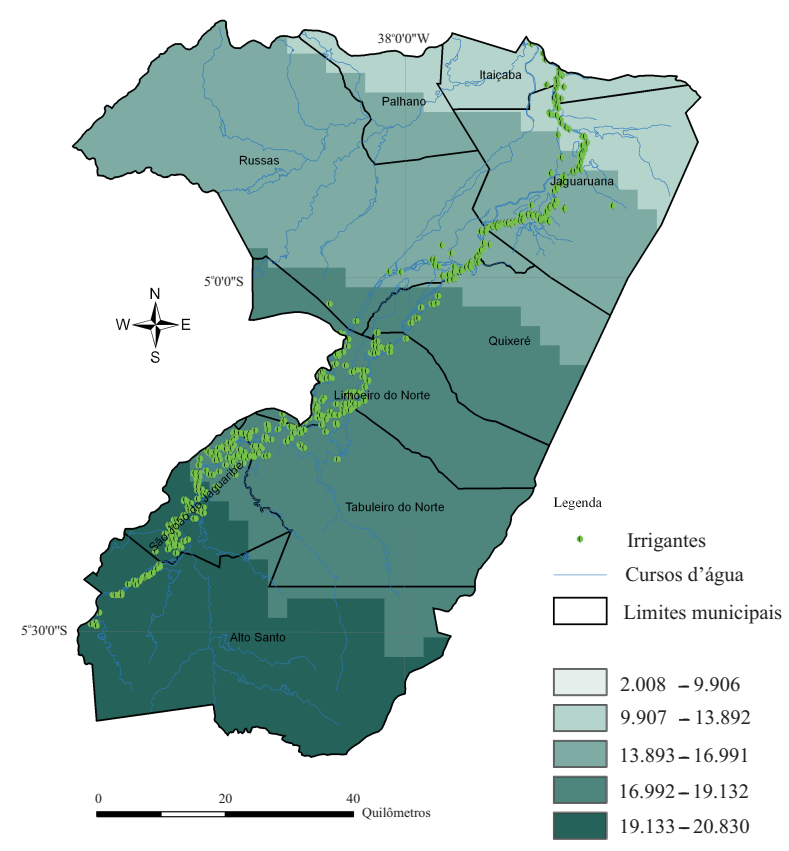

Figura 3. Mapa temático da necessidade hídrica bruta $\left(\mathrm{m}^{3} \mathrm{ha}^{-1}\right)$ anual em 2040, no cenário B2. 


\section{Conclusões}

1. As mudanças climáticas, conforme o modelo e cenários utilizados, impactam na demanda de água para irrigação das culturas perenes, como conseqüência do balanço hídrico, determinado pela evapotranspiração e pela precipitação.

2. Temperaturas mais elevadas causam maior demanda para evapotranspiração das plantas, com tendência de ocorrer aumento na demanda de água para irrigação, agravada pela redução da precipitação.

3. A mudança climática funciona como fonte de aumento na demanda de água para a agricultura, com a necessidade de expansão da área irrigada.

\section{Referências}

ALLEN, R.G.; PEREIRA, L.S.; RAES, D.; SMITH, M. Crop evapotranspiration: guidelines for computing crop water requirements. Rome: FAO, 1998. 300p. (FAO. Irrigation and Drainage Paper, 56).

BERNARDO, S.; SOARES, A.A.; MANTOVANI, E.C. Manual de irrigação. 7.ed. Viçosa: Editora da UFV, 2005. 611p.

BINDER, L.C.W. Climate change and watershed planning in Washington State. Journal of the American Water Resources Association, v.42, p.915-926, 2006.

COGERH. Companhia de Gestão de Recursos Hídricos. Cadastro de Outorga: 2008. Fortaleza, 2008. 1 CD-ROM.

COSTA, R.N.T.; SOUZA, F. de. Irrigação por superfície. In: ROSA, M. de F.; GONDIM, R.S.; FIGUEIRÊDO, M.C.B. (Ed.). Gestão Sustentável no Baixo Jaguaribe, Ceará. Fortaleza: Embrapa Agroindústria Tropical, 2006. p.261-288.

DÍAZ， J.A.R.; WEATHERHEAD， E.K.; KNOX, J.W.; CAMACHO, E. Climate change impacts on irrigation water requirements in the Guadalquivir river basin in Spain. Regional Environmental Change, v.7, p.149-159, 2007.

DIBIKE, Y.B.; COULIBALY, P. Hydrologic impact of climate change in the Saguenay watershed: comparison of downscaling methods and hydrologic models. Journal of Hydrology, v.307, p.145-163, 2005.

DÖLL, P.; HAUSCHILD, M. Model-based regional assessment of water use: an example for semi-arid Northeastern Brazil. Water International, v.27, p.310-320, 2002.

DOORENBOS, J.; PRUITT, W.O. Guidelines for predicting crop water requirements. Rome: FAO, 1977. 179p. (FAO. Irrigation and Drainage Paper, 24).

EITZINGER, J.; STASTNÁ, M.; ZALUD, Z.; DUBROVSKY, M. A simulation study of the effect of soil water balance and water stress on winter wheat production under different climate change scenarios. Agricultural Water Management, v.61, p.195-217, 2003.
FISCHER, G.; TUBIELlO, F.N.; VELTHUIZEN, H.V.; WIBERG, D.A. Climate change impacts on irrigation water requirements: effects of mitigation: 1990-2008. Technological Forecasting \& Social Change, v.74, p.1083-1107, 2007.

FORTES, P.S.; PLATONOV, A.E.; PEREIRA, L.S. Gisareg-A GIS based irrigation scheduling simulation model to support improved water use. Agricultural Water Management, v.77, p.159-179, 2005.

JOHNS, T.C.; CARNELL, R.E.; CROSSLEY, F.F.; MITCHELL, J.F.B.; SENIOR, C.A.; TETT, S.F.B.; WOOD, R.A. The second Hadley Centre coupled ocean-atmosphere GCM: model description, spinup and validation. Climate Dynamics, v.13, p.103-134, 1997.

JOHNS, T.C.; GREGORY, J.M.; IGRAM, W.J.; JOHNSON, C.E.; JONES, A.; LOWE, A.; MITCHELL, J.F.B.; ROBERTS, D.L.; SEXTON, D.M.H.; STEVESON, D.S.; TETT, S.F.B.; WOODAGE, M.J. Anthropogenic climate change for 1860 to 2100 simulated with the HadCM3 model under updated emissions scenarios. Climate Dynamics, v.20, p.583-612, 2003.

JONES, R.G.; NOGUER, M.; HASSELL, D.C.; HUDSON, D.; WILSON, S.S.; JENKINS, G.J.; MITCHELL, J.F.B. Generating high resolution climate change scenarios using Precis. Exeter: Meteorological Office Hadley Centre, 2004. 39p.

KNOX, J.W.; WEATHERHEAD, E.K.; BRADLEY, R.I. Mapping the total volumetric irrigation water requirements in England and Wales. Agricultural Water Management, v.33, p.1-18, 1997.

KROL, M.S.; BRONSTERT, A. Regional integrated modeling of climate change impacts on natural resources and resources usage in semi-arid Northeast Brazil. Environmental Modelling \& Software, v.22, p.259-268, 2007.

KROL, M.S.; JAEGER, A.; BRONSTERT, A.; GÜNTNER, A. Integrated modeling of climate change, water, soil, agricultural and social-economic processes: a general introduction of the methodology and some exemplary results from the semi-arid Northeast of Brazil. Journal of Hydrology, v.328, p.417-431, 2006.

MEDEIROS, Y.D.P. Análise dos impactos das mudanças climáticas em região semi-árida. Revista Brasileira de Recursos Hídricos, v.8, p.127-136, 2003.

MOHAN, S. Intercomparison of evapotranspiration estimates. Journal des Sciences Hydrologiques, v.36, p.447-460, 1991.

NAKICENOVIC, N.; ALCAMO, J.; DAVIS, G.; DE VRIES, B.; FENHANN, J.; GAFFIN, S.; GREGORY, K.; GR, A.; JUNG, T.Y.; KRAM, T.; LA ROVERE, E.L.; MICHAELIS, L.; MORI, S.; MORITA, T.; PEPPER, W.; PITCHER, H.; PRICE, L.; RIAHI, K.; ROEHRL, A.; ROGNER, H.H.; SANKOVSKI, A.; SCHLESINGER, M.; SHUKLA, P.; SMITH, S.; SWART, R.; VAN ROOIJEN, S.; VICTOR, N.; DADI, Z. Special report on emission scenarios. Intergovernmental Panel on Climate Change, 2000. Disponível em: <http://www.ipcc.ch/ipccreports/sres/emission/ index.htm>. Acesso em: 3 jan. 2007.

NUNES, A.B. de A. Avaliação ex-post da sustentabilidade hídrica e da conservação ambiental de perímetros irrigados implantados: o caso do Perímetro Irrigado Jaguaribe-Apodi (Dija). 2006. 176p. Tese (Doutorado) - Universidade Federal do Ceará, Fortaleza. 
ROECKNER, E.; ARPE, K.; BENGTSSON, L.; CHRISTOPH, M.; CLAUSSEN, M.; DÜMENIL, L.; ESCH, M.; GIORGETTA, M.; SCHLESE, U.; SCHULZWEIDA, U. The atmospheric general circulation model ECHAM4: Model description and simulation of present-day climate. Hamburgo: Max Planck Institut für Meteorologie, 1996. 90p. (Max Planck Institut für Meteorologie Report, 218).

SAMANI, Z.A.; PESSARAKLI, M. Estimating potential crop evapotranpiration with minimum data in Arizona. Transactions of the ASAE, v.29, p.522-524, 1986.

SCHLENKER, W.; HANEMANN, W.M.; FISHER, A.C. Water availability, degree days and the potential impact of climate change on irrigated agriculture in California. Climatic Change, v.81, p.19-38, 2007.

SILVA, C.S.; WEATHERHEAD, E.K.; KNOX, J.W.; DÍAZ, J.A.R. Predicting the impacts of climate change: a case study of paddy irrigation water requirements in Sri Lanka. Agricultural Water Management, v.93, p.19-29, 2007.

TSANIS, I.K.; NAOUM, S. The effect of spatially distributed meteorological parameters on irrigation water demand assessment. Advances in Water Resources, v.26, p.311-324, 2003.

TUBIELLO, F.N.; EWERT, F. Stimulating the effects of elevated $\mathrm{CO}_{2}$ on crops: approaches and applications for climate change. European Journal of Agronomy, v.18, p.57-74, 2002.

VICTORIA, F.B.; VIEGAS FILHO, J.S.; PEREIRA, L.S.; TEIXEIRA, J.L.; LANA, A.E. Multi-scale modeling for water resources planning and management in rural basins. Agricultural Water Management, v.77, p.4-20, 2005.

WILBY, R.L.; DAWSON, C.W.; BARROW, E.M. SDSM: a decision support tool for the assessment of regional climate change impacts. Environmental Modelling \& Software, v.17, p.147-159, 2002.

Recebido em 23 de setembro de 2008 e aprovado em 19 de novembro de 2008 